\title{
Modifiable and Nonmodifiable Predictive Factors Associated with the Outcomes of Total Knee Arthroplasty
}

\author{
Davide E. Bonasia ${ }^{1}$ Anna Palazzolo ${ }^{1}$ Umberto Cottino ${ }^{1}$ Francesco Saccia ${ }^{2}$ Claudio Mazzola ${ }^{3}$ \\ Federica Rosso ${ }^{1}$ Roberto Rossi ${ }^{1}$
}

${ }^{1}$ Department of Orthopaedics and Traumatology, AO Ordine Mauriziano Hospital, University of Torino, Torino, Italy

2 Ospedale Torino Nord Emergenza San Giovanni Bosco, SC Ortopedia e Traumatologia, Torino, Italy

${ }^{3}$ Ospedali Galliera Genova, SC Ortopedia delle articolazioni, Genoa, Italy

Joints 2019;7:13-18.

\author{
Address for correspondence Davide E. Bonasia, MD, Department of \\ Orthopaedics and Traumatology, AO Ordine Mauriziano Hospital, \\ University of Torino, Via Lamarmora 26, 10128 Torino, Italy \\ (e-mail: davidebonasia@virgilio.it).
}

\author{
Abstract \\ Keywords \\ - total knee \\ arthroplasty \\ - predictive factor \\ - prognostic factor \\ - outcome \\ - patient optimization
}

Total knee arthroplasty (TKA) is a valuable treatment option for advanced osteoarthritis in patients unresponsive to conservative treatments. Despite overall satisfactory results, the rate of unsatisfied patients after TKA remains high, ranging from 5 to $40 \%$. Different modifiable and nonmodifiable prognostic factors associated with TKA outcomes have been described. The correction, whenever possible, of modifiable factors is fundamental in preoperative patients' optimization protocols. Nonmodifiable factors can help in predicting the outcomes and creating the right expectations in the patients undergoing TKA. The goal of this review is to summarize the modifiable and nonmodifiable prognostic factors associated with TKA outcomes.

\section{Introduction}

Knee osteoarthritis (OA) may occur due to a wide variety of factors (e.g., posttraumatic, genetic, metabolic, biomechanical). ${ }^{1,2}$ Total knee arthroplasty (TKA) is generally accepted as a valuable treatment for advanced $O A$ in patients unresponsive to conservative treatments. Despite overall satisfactory results, the estimated prevalence of patients reporting minor or no improvement of symptoms and pain after TKA remains high, ranging from 5 to $40 \%{ }^{3}$

Vince identified nine causes of failure for TKA including: (1) aseptic loosening; (2) tibial femoral instability; (3) patellar complications and malrotation; (4) no diagnosis, the socalled mystery knee; (5) structural failure of the implant; (6) infection; (7) extensor mechanism rupture; (8) stiffness; and (9) fracture. ${ }^{4}$ Technical errors are still a leading cause of failure and TKAs should be performed in high volume centers. ${ }^{5,6}$ A significant association between low surgeon volume and higher rate of infection (0.26-2.8\% higher), procedure time (165 vs. 135 minutes), longer length of stay (0.4-2.13 days longer), transfusion rate (13 vs. $4 \%$ ), and worse patient reported outcomes was found. ${ }^{7}$ However, the rate of patients unsatisfied with their TKA is high also in high volume centers. A multicenter study, performed by an independent, blinded third party survey center, quantified the degree of patient satisfaction and residual symptoms in TKA patients. A total of $90 \%$ of patients were satisfied with the overall function of the knee, only $66 \%$ felt their knee was normal, with a reported incidence of residual symptoms and functional problems ranging from 33 to $54 \% .^{8}$

For this reason, there is a great interest in identifying prognostic/predictive factors significantly associated with the outcomes of TKA. These factors can be divided in modifiable and nonmodifiable. The correction, whenever possible, of modifiable factors is the cornerstone of preoperative patients' optimization protocols. Nonmodifiable factors can received

September 12, 2017

accepted

January 3, 2019

published online

February 1, 2019
DOI https://doi.org/

10.1055/s-0039-1678563. ISSN 2282-4324.
Copyright (C 2019 Georg Thieme Verlag KG Stuttgart · New York
License terms

(요 (1) $\Theta$ 
help predicting the outcomes and creating the right expectations in patients undergoing TKA. The goal of this review, based on the recent literature, is to summarize the modifiable and nonmodifiable prognostic factors associated with TKA outcomes.

\section{Nonmodifiable Prognostic Factors}

Female sex and young age were found to be associated with worse outcomes and persistent postoperative pain., ${ }^{3,9}$ In a national multicenter study, the degree of residual symptoms and specific functional deficits in 661 young patients (average age, 54 years) was quantified at 1 to 4 years after primary TKA. Overall, $91 \%$ of the patients were satisfied with their pain relief. After recovery, $47 \%$ reported complete absence of a limp and $50 \%$ had participated in their recreational activity in the past 30 days. However, about one-third of the patients reported residual symptoms and limitations after modern TKA. $^{9}$

Income and socioeconomic factors have also been identified as prognostic factors. Barrack et al noted that patients reporting incomes $<25,000$ USD were less likely to be satisfied with TKA outcomes and more likely to have functional limitations than patients with higher incomes. ${ }^{10}$ Similarly, Judge et al, in a study from the Elective Orthopaedic Centre database (1,991 patients receiving primary TKA in southwest London from 2005-2008) noted that patients living in poorer areas had worse outcomes. ${ }^{11}$

In the previously cited paper, Judge et al also noted that patients with less severe preoperative disease (less preoperative pain and better function) obtained the best outcomes compared with those with more advanced disease. ${ }^{11}$

When considering the initial diagnosis leading to a TKA, patients with rheumatoid arthritis did better than those with $\mathrm{OA}$, in terms of pain outcomes. ${ }^{11}$

\section{Modifiable Prognostic Factors}

Different modifiable factors have been associated with increased risk of: (1) infection, (2) revision, (3) patient readmission, and (4) poor outcomes. Reducing these factors, whenever possible, during the preoperative phase, can decrease the number of complications and unsatisfied patients.

Kee et al conducted a retrospective review of all revision total hip and knee arthroplasties performed at an academic, tertiary, referral center within 2 years of primary surgery. The presence of body mass index (BMI) $>40$, hemoglobin A1c $>8$, poor dentition, and smoking status were recorded. Risk factors were described and compared between infected and noninfected revisions. Out of a total of 128 revision arthroplasties, 23 of 57 (40.4\%) total hip revisions and 31 of $71(43.7 \%)$ total knee revisions had at least one modifiable risk factor. Infected TKA patients were more likely to smoke, have poor dentition, and have $>1$ contraindication compared with noninfected patients. The authors concluded that a high percentage of patients undergoing early revision arthroplasty had at least one modifiable risk factor for a primary joint arthroplasty, and that these factors are need to be reduced through counseling and appropriate referral for patient optimization. ${ }^{12}$

Iorio and Osmani retrospectively reviewed a consecutive series of 3,836 primary and revision TKAs performed at a single institution from 2009 to 2011. Forty-one (1.07\%) deep surgical site infections were identified. Infection developed in 26 of $3,419(0.76 \%)$ primary cases and 15 of 417 (3.6\%) revision cases $(p=0.001)$. Multivariate analysis revealed significant risk factors for infection. For primary TKA, these included: female gender (OR [odds ratio] $=3.3$ ), tobacco use $(\mathrm{OR}=3.1)$, pulmonary disease $(\mathrm{OR}=5.3)$, Staphylococcus aureus colonization $(\mathrm{OR}=4)$. For revision TKA, these included: diabetes mellitus (DM) complications $(\mathrm{OR}=15)$ and not-same-day surgery $(\mathrm{OR}=3.8)$. For these reasons, different institutions implemented programs to optimize patients before total joint replacement. The New York University School of Medicine implemented a program to modify seven risk factors for infection including: (1) methicillinresistant S. aureus (MRSA) screening and decolonization, weight-based antibiotic dosing, and use of vancomycin and gentamycin in high-risk patients; (2) hepatitis-C screening and treatment, HIV optimization; (3) smoking cessation (hard stop); (4) aggressive weight control (hard stop at a BMI of 40); (5) drug and alcohol interventions; (6) DM control and nutritional interventions (hard stop with glucose 0.180); (7) screening for high-risk patients with venous thromboembolic disease. ${ }^{13}$

Many predictive risk factors for readmission have been described, including: black race, discharge to inpatient rehabilitation, increased duration of hospital stay, advanced age, respiratory conditions, increased BMI, diabetes, ASA (American Society of Anesthesiologists) grade $>2$, increased operative time, cardiovascular problems, venous thromboembolic disease, smoking status, behavioral or neurocognitive problems, drug and alcohol abuse, physical deconditioning, and fall risk. ${ }^{14}$ Factors such as age, race, and ASA grade are not modifiable. The modifiable risk factors can be inserted in models specifically designed to calculate the risk of readmission (readmission risk assessment tool or RRAT). With these tools, surgery can be delayed when the score is too high, until patient's medical optimization has been achieved. ${ }^{14}$

\section{Hepatitis-C and Cirrhosis}

Issa et al in a matched control study on the nationwide inpatient sample database identified patients who underwent total hip arthroplasty (THA) or TKA in the United States from 1998 to 2010. Controls were matched in a three-to-one ratio to patients with hepatitis- $C$ infection according to surgical procedure, age, race, sex, Deyo's comorbidity score, and year of surgical procedure. Out of 1,700,400 total joint arthroplasties (TJA), 8,044 patients $(0.47 \%)$ had a documented hepatitis-C infection. Patients with hepatitis-C had a 30\% increased risk of any complication, a $15 \%$ increased risk of a medical complication, a $78 \%$ increased risk of a surgical complication, and a mean length of stay that was $14 \%$ longer. $^{15}$ 
Tiberi et al, in a retrospective case control study regarding THA and TKA (115 patients per group), reported that patients with cirrhosis had prolonged length of stay, higher rates of discharge to nursing facilities, readmission in 90 days, urinary tract infections $(p<0.01)$, renal failure $(p=0.03)$, blood transfusions $(p<0.01)$, gastrointestinal hemorrhage $(p=0.04)$, dislocations $(p=0.01)$, infections $(p=0.02)$, and revisions $(p=0.04)$ within 90 days, compared with matched control subjects. One-year $(p=0.01)$ and longterm $(p=0.0002)$ mortality rates were also higher in cirrhosis patients. A model for end-stage liver disease (MELD) score of 10 or greater predicted a three times increased likelihood of any complication and 4.1 times increased likelihood of death. The authors concluded that patients with cirrhosis undergoing THA and TKA should be counseled regarding their increased risk of medical complications, surgical complications, and death. ${ }^{16}$

Similar results were reported by Jiang et al in a study based on national and state-level databases, including patients with and without liver cirrhosis who underwent TJA. From 2000 to 2010, 16,634 patients with cirrhosis underwent TJA. Periprosthetic joint infections were more common after TKA in patients with cirrhosis (2.7 vs $0.8 \%$; HR [hazard ratio] $=3.4 ; p<0.001)$. The number of TKA surgeries increased faster for patients with cirrhosis than for patients without cirrhosis ( 213 vs. $128 \%$; $p<0.001$ ). Patients with cirrhosis had higher risk of infection, longer length of hospital stay, increased costs, and higher rates of mortality, readmission, and reoperation. ${ }^{17}$

\section{Obesity and Malnutrition}

Obesity is one of the most important risk factors for knee OA and BMI $>30$ is significantly associated with knee OA (OR $=2.81) .{ }^{18}$ Obesity is not only common in TKA patients, but is also an established risk factor for complications after TKA. Obese patients are more prone to have medical (thromboembolic, cardiovascular and respiratory events) and anesthesiologic (ventilation and intubation) complications together with a technically more demanding surgery (in terms of exposure, alignment, and tibial fixation). ${ }^{19}$ Multiple studies have shown worse TKA survivorship in the obese population. Abdel et al reported that patients with a BMI $>35$ had a significantly increased risk of tibial loosening $(p=0.05) .{ }^{20}$ In addition, morbidly obese patients with TKA have a $43.9 \%$ increase risk of revision TKA compared with a normal BMI group (18.5-25). ${ }^{21}$ Wallace et al in a study on 32,485 TKA patients described that increased BMI was associated with a significantly higher risk of wound infections (from 3-4.1\%, $p<0.05)$, thromboembolic events $(2.0-3.3 \%, p<0.01)$, compared with nonobese patients. ${ }^{22}$ Similarly, in a prospective study of 1,214 consecutive TKAs, Dowsey and Choong noted an overall infection rate of $1.5 \%(n=18)$. The deep prosthetic infection rate in the morbidly obese cohort (WHO [World Health Organization] classes I-III) was significantly greater than the rate in the nonobese cohort $(\mathrm{OR}=8.96){ }^{23}$

Although BMI has been considered for a long time the main obesity index in patients undergoing TKA, recently other parameters have shown to better correlate with TKA outcomes/complications in the obese population. Watts et al hypothesized that distances patella-skin (prepatellar thickness) and tibial tubercle-skin (pretubercular thickness) measured on routine lateral knee radiographs were associated with outcomes of TKA. Intraobserver and interobserver reliabilities were excellent for both measurements. The authors noted that patients who underwent TKA revision had significantly greater prepatellar $(p=0.0001)$ and pretubercular ( $p=0.0006)$ soft tissue thickness compared with the control group. Prepatellar thickness $\geq 15 \mathrm{~mm}$ and pretubercular thickness $\geq 25 \mathrm{~mm}$ increased the risk of early reoperation by $2.0 \times(p=0.0003)$ and $1.6 \times(p=0.023)$, respectively, and were more predictive measurements than BMI. ${ }^{24}$ Ledford et al studied percent body fat as a predictive measurement of function after TJA. Percent body fat is total fat mass divided by total weight and represents a patient-specific measure that does distinguish muscle from fat mass. In a prospective study on 215 patients undergoing TKA or THA, the authors found that higher percent body fat predicted occurrence of any medical or surgical complication, University of California at Los Angeles activity score, and pain scores, whereas BMI was not predictive of these functional outcomes. Additionally, percent body fat was predictive of outcomes after TKA (Knee injury and Osteoarthritis Outcome Score (KOOS) pain and KOOS activities of daily living) but not for those after THA. ${ }^{25}$

Recently, malnutrition (defined as hypoalbuminemia, albumin $<3.5 \mathrm{~g} / \mathrm{dL}$ ) has shown to be a better predictor than obesity for complications after TKA. Fu et al in a study on 34,800 TKAs, showed that malnutrition prevalence increased with BMI (6.1\% in BMI > 40 vs. $3.7 \%$ in nonobese) and that hypoalbuminemia was a stronger and more consistent independent risk factor, for any complication, wound, cardiac, or respiratory complications, and death than BMI. The authors concluded that strategies for medical optimization of these conditions should be implemented. ${ }^{26}$ Similarly, Walls et al showed that hypoalbuminemia was a significant risk factor for mortality (5.94-fold risk of 30-day mortality) and major morbidity among THA patients, while morbid obesity was only associated with an increased risk of superficial surgical site infection. ${ }^{27}$ Yi et al in a study on 600 revision TJAs evaluated the association between nutritional parameters (including serum albumin, total lymphocyte count, and transferrin) and septic failure or acute infection after revision TJA. Most patients (53\%) revised for infected TJA had one or more laboratory parameters suggestive of malnutrition, compared with $33 \%$ of revisions for a noninfectious etiology. Patients who were of normal weight at the time of revision had the highest frequency of malnutrition parameters (51\%), compared with obese patients (32\%). Among the 375 aseptic revisions, 12 developed an acute postoperative infection (3\%). Having laboratory parameters suggestive of malnutrition was independently associated with both chronic TJA infection $(p=0.003$; OR $=2.1)$ and acute postoperative infection complicating an aseptic revision $(p=0.02 ;$ OR $=5.9){ }^{28}$

It has to be mentioned here that the role of bariatric surgery as a tool for patients' optimization before TKA is controversial. 
Werner et al evaluated three large cohorts, 90 days after TKA: nonobese patients $(n=66,523)$, morbidly obese patients (WHO class III) who did not undergo bariatric surgery $(n=11294)$, and morbidly obese patients (WHO class III) who underwent bariatric surgery before TKA $(n=219)$. The authors found reduced rates of both major complications $(\mathrm{OR}=0.45 ; p=0.001)$ and minor complications $(\mathrm{OR}=0.61$; $p=0.01$ ) in the bariatric cohort compared with the morbidly obese cohort who did not undergo bariatric surgery. 19,29 Martin et al identified a cohort of 91 patients who had undergone bariatric surgery before TKA (mean BMI before bariatric surgery, $51.1 \mathrm{~kg} / \mathrm{m}^{2}$; mean BMI at the time of TKA, $37.3 \mathrm{~kg} / \mathrm{m}^{2}$ ). This cohort was then matched with two groups that did not undergo bariatric surgery before TKA; a cohort matched to the bariatric cohorts prebariatric BMI of $51 \mathrm{~kg} / \mathrm{m}^{2}$ before TKA (1:1 match; $n=91)$ and a cohort matched to the bariatric cohorts postbariatric BMI of $37 \mathrm{~kg} / \mathrm{m}^{2}$ before TKA ( $1: 2$ match; $n=182$ ). The bariatric cohort had a higher risk of reoperation $(\mathrm{HR}=2.6 ; p=0.02)$ than did the high BMI control cohort, and higher risk of reoperation $(\mathrm{HR}=2.4 ; p=0.2)$ and revision $(\mathrm{HR}=2.2 ; p=0.04)$ than did the lower BMI cohort. One possible explanation for worse outcomes in bariatric patients undergoing TKA is concomitant malnutrition in this population. ${ }^{19,30}$

\section{Diabetes Mellitus}

Diabetes mellitus (DM) is an established risk factor for infections but evidence is conflicting to what extent perioperative hyperglycemia, glycemic control, and treatment around the time of surgery modify the risk of prosthetic joint infections (PJIs). In a cohort of 20,171 THA and TKA, Maradit Kremers et al observed a significantly higher risk of PJIs among patients with DM $(\mathrm{HR}=1.55)$, patients using diabetes medications ( $\mathrm{HR}=1.56)$, and patients with perioperative hyperglycemia $(H R=1.59)$, but the effects were attenuated after adjusting for BMI, type of surgery, ASA score, and operative time. Although data were limited, there was no association between hemoglobin A1c values and PJIs. ${ }^{31}$ Marchant et al identified, from the nationwide inpatient sample, over 1 million patients who underwent TJA. These patients were divided into three groups: (1) uncontrolled $\operatorname{DM}(n=3,973),(2)$ controlled DM ( $n=105,485)$, and (3) no $\operatorname{DM}(n=920,555)$. The groups were compared with regard to common surgical and systemic complications, mortality, and hospital course alterations. Additional stratification compared the effects of glucose control among patients with type-I and type-II DM. Compared with patients with controlled DM, patients with uncontrolled DM had a significantly increased odds of stroke $(\mathrm{OR}=3.42)$, urinary tract infection $(\mathrm{OR}=1.97)$, ileus $(\mathrm{OR}=2.47)$, postoperative hemorrhage $(\mathrm{OR}=1.99)$, transfusion $(\mathrm{OR}=1.19)$, wound infection $(\mathrm{OR}=2.28)$, and death $(\mathrm{OR}=3.23) .{ }^{32}$ Watts et al compared the outcomes between nondiabetic $(n=1,284)$, type II diabetic $(n=530)$, and insulin-dependent type II diabetic $(n=164)$ morbidly obese (BMI $\left.\geq 40 \mathrm{~kg} / \mathrm{m}^{2}\right)$ patients undergoing primary TKA at 6-year follow-up. Patients with type II diabetes mellitus (DM) had similar outcomes when compared with non-DM patients. However, patients with insulin dependence had an increased risk of reoperation $(\mathrm{HR}=1.8)$, revision $(\mathrm{HR}=2)$, PJI $(\mathrm{HR}=2.1)$, decreased 10 -year implant survivorship (84 vs. $92 \%$ ) when compared with non-DM patients. ${ }^{33}$

\section{Vitamin D Deficiency}

Recently the role of vitamin D deficiency in preoperative joint function and TKA results has been studied. Jansen and Haddad studied the plasma 25(OH)D3 levels measured in a group of 139 elderly patients with advanced OA. In 33 patients (24\%), a vitamin D deficiency $(<40 \mathrm{nmol} / \mathrm{L})$ was found and in all cases, it was associated with a secondary hyperparathyroidism. The mean preoperative Knee Society Score (KSS) was significantly lower in the vitamin D deficient group than in the vitamin D sufficient group. ${ }^{34}$

The influence of vitamin $D$ on functional performance before and after TKA was retrospectively reviewed by Maniar and colleagues. Out of 120 patients, 64 had vitamin D deficiency $(25(\mathrm{OH}) \mathrm{D} 3<30 \mathrm{ng} / \mathrm{mL})$ preoperatively. All 120 patients received vitamin $\mathrm{D}$ oral supplementation postoperatively. Preoperative function was significantly lower in patients with vitamin D deficiency (Western Ontario and McMaster Universities Osteoarthritis score), but at 3 months, all functional scores were similar. The authors concluded that TKA should not be delayed in vitamin $\mathrm{D}$ deficient patients and supplementation in the postoperative period is preferable. ${ }^{35}$

Maier et al measured serum 25(OH)D levels after admission of every patient scheduled to receive TJA (109 patients). Additionally, serum 25(OH)D levels were measured for every patient presenting with PJI $(n=50)$ or aseptic loosening $(n=31)$. The prevalence of normal $(>30 \mathrm{ng} / \mathrm{mL})$, insufficient (20-30 ng/mL), and deficient $(<20 \mathrm{ng} / \mathrm{mL}) 25(\mathrm{OH}) \mathrm{D}$ levels was determined. All tested patient subgroups showed low 25 (OH)D levels. Patients with PJI had significantly lower 25(OH) D levels than patients scheduled for primary arthroplasty $(p<0.001)$. A significant difference was also found between patients with PJI and patients with aseptic loosening. ${ }^{36}$

\section{Anxiety and Depression}

Psychiatric illnesses have been identified as significant predictive factors for complications and outcomes after TKA and many authors advise appropriate preoperative counseling. Rasouli et al, out of 1,969 THA and TKA patients, described a 12.7\% (TKA) and 6.4\% (THA) prevalence of depression or anxiety. Anxiety and depression and higher ASA score were independent predictors of complications. ${ }^{37}$ Browne et al assessed the incidence of the diagnosis of depression and determined the impact of this diagnosis on early postoperative outcomes following TJA (497,222 implants). The prevalence of depression in the arthroplasty population was 10.0\%. Patients with depression were significantly more likely to be white, female, and have Medicaid as a primary payer. Depression was associated with a greater risk of postoperative psychosis ( $\mathrm{OR}=1.74)$, anemia $(\mathrm{OR}=1.14)$, infection $(\mathrm{OR}=1.33)$, and pulmonary embolism (OR $=1.20)$, and a lower risk of cardiac $(\mathrm{OR}=0.93)$, and gastrointestinal complications $(\mathrm{OR}=0.80) .{ }^{38}$ Out 186 patients operated with a primary TKA, Ali et al described a $15 \%$ rate 
of patients dissatisfied or uncertain with the result of their TKA 4 years postoperatively. Of the dissatisfied patients, 59\% had reported anxiety/depression preoperatively compared with $7 \%$ in the satisfied or very satisfied groups. Patients with preoperative anxiety or depression had more than six times higher risk to be dissatisfied compared with patients with no anxiety or depression. ${ }^{39}$

Singh and Lewallen studied 7,139 primary TKAs at 2 years, 4,234 at 5 years, 1,533 revision TKAs at 2 years, and 881 at 5 years. The authors found that depression was associated with significantly lower odds of 0.5 of "much better" knee functional status (relative to same or worse status) 2 years after primary TKA. A higher Deyo-Charlson index for medical comorbidities was significantly associated with lower odds of 0.5 of "much better" knee functional status after revision TKA for every five-point increase in score. ${ }^{40}$

Klement et al noted that the risk of complications following total joint arthroplasty is higher among patients with other psychiatric illnesses. The authors showed that patients with a diagnosis of depression, bipolar disorder, or schizophrenia had an increased likelihood of knee extensor mechanism rupture $(\mathrm{OR}=2.18)$, periprosthetic fracture ( $O R$ $=2.2)$, prosthetic join infection $(\mathrm{OR}=2.08)$, and need for revision arthroplasty $(\mathrm{OR}=2.01)$ at minimum of 2-year follow-up. ${ }^{41,42}$

\section{Metal Hypersensitivity}

The exact role of metal allergy/hypersensitivity is still unknown in painful TJA. It is uncertain whether delayedtype hypersensitivity reactions against implanted metals contribute to osteolysis. A recent review showed that some cytokines produced by T-helper cells (i.e., Interleukin- 2 and interferon gamma) are expressed in metal allergy and frequently identified in histological samples from TJA patients. ${ }^{43,44}$ Granchi et al showed that the prevalence of metal allergy is higher after TJA ( $O R=1.5)$ when compared with controls, and that the prevalence is even higher when patients with failed TJA are compared with patients with stable TJA $(\mathrm{OR}=2.8) .{ }^{45}$ However, it is still unknown, whether preoperative metal allergy may be a risk factor for revision surgery or whether it is a result of TJA failure with secondary sensitization. ${ }^{44}$ Caicedo et al showed that in a selected group of patients ( 1,038 men and 1,575 women) with painful, aseptic TJA who were referred for metalsensitivity testing, females exhibited a higher level of pain and demonstrated a higher rate and severity (as measured by lymphocyte stimulation index) of metal sensitization compared with males. ${ }^{46}$

Münch et al retrieved, from Danish databases, all TKAs and revision TKAs with a positive contact allergy patch test (327 patients). The prevalence of contact allergy to nickel, chromium, and cobalt was comparable in patients with and without revision surgery. However, in patients with two or more episodes of revision surgery, the prevalence of cobalt and chromium allergy was markedly higher. Metal allergy that was diagnosed before implant surgery appeared not to increase the risk of implant failure and revision surgery. ${ }^{44}$

\section{Conclusion}

A better understanding of nonmodifiable negative prognostic factors helps the surgeon in creating correct expectations in patients undergoing TKA. On the other hand, a precise knowledge of modifiable predictive factors is fundamental in implementing preoperative optimization protocols for TKA patient with the goal of reducing complications, revision rate, costs, and unsatisfactory outcomes. Different patient optimization protocols are already available in the literature. $^{14}$

Conflict of Interest

None declared.

\section{References}

1 Deveza LA, Melo L, Yamato TP, Mills K, Ravi V, Hunter DJ. Knee osteoarthritis phenotypes and their relevance for outcomes: a systematic review. Osteoarthritis Cartilage 2017;25(12): 1926-1941

2 Rossi R, Bonasia DE, Blonna D, Assom M, Castoldi F. Prospective follow-up of a simple arthroscopic-assisted technique for lateral tibial plateau fractures: results at 5 years. Knee 2008;15(05): 378-383

3 Sakellariou VI, Poultsides LA, Ma Y, Bae J, Liu S, Sculco TP. Risk assessment for chronic pain and patient satisfaction after total knee arthroplasty. Orthopedics 2016;39(01):55-62

4 Vince KG. Why knees fail. J Arthroplasty 2003;18(03, Suppl 1 39-44

5 Lombardi AV Jr, Berend KR, Adams JB. Why knee replacements fail in 2013: patient, surgeon, or implant? Bone Joint J 2014;96-B (11, Suppl. A):101-104

6 Rossi R, Bruzzone M, Bonasia DE, Marmotti A, Castoldi F. Evaluation of tibial rotational alignment in total knee arthroplasty: a cadaver study. Knee Surg Sports Traumatol Arthrosc 2010;18(07): 889-893

7 Lau RL, Perruccio AV, Gandhi R, Mahomed NN. The role of surgeon volume on patient outcome in total knee arthroplasty: a systematic review of the literature. BMC Musculoskelet Disord 2012; 13:250

8 Nam D, Nunley RM, Barrack RL. Patient dissatisfaction following total knee replacement: a growing concern? Bone Joint J 2014;96B(11, Suppl. A):96-100

9 Parvizi J, Nunley RM, Berend KR, et al. High level of residual symptoms in young patients after total knee arthroplasty. Clin Orthop Relat Res 2014;472(01):133-137

10 Barrack RL, Ruh EL, Chen J, et al. Impact of socioeconomic factors on outcome of total knee arthroplasty. Clin Orthop Relat Res 2014;472(01):86-97

11 Judge A, Arden NK, Cooper C, et al. Predictors of outcomes of total knee replacement surgery. Rheumatology (Oxford) 2012;51(10): 1804-1813

12 Kee JR, Mears SC, Edwards PK, Barnes CL. Modifiable risk factors are common in early revision hip and knee arthroplasty. J Arthroplasty 2017;32(12):3689-3692

13 Iorio R, Osmani FA. Strategies to prevent periprosthetic joint infection after total knee arthroplasty and lessen the risk of readmission for the patient. J Am Acad Orthop Surg 2017;25 (Suppl 1):S13-S16

14 Boraiah S, Joo L, Inneh IA, et al. Management of modifiable risk factors prior to primary hip and knee arthroplasty: a readmission risk assessment tool. J Bone Joint Surg Am 2015;97(23): 1921-1928 
15 Issa K, Boylan MR, Naziri Q, Perfetti DC, Maheshwari AV, Mont MA. The impact of hepatitis $C$ on short-term outcomes of total joint arthroplasty. J Bone Joint Surg Am 2015;97(23):1952-1957

16 Tiberi JV III, Hansen V, El-Abbadi N, Bedair H. Increased complication rates after hip and knee arthroplasty in patients with cirrhosis of the liver. Clin Orthop Relat Res 2014;472(09): 2774-2778

17 Jiang SL, Schairer WW, Bozic KJ. Increased rates of periprosthetic joint infection in patients with cirrhosis undergoing total joint arthroplasty. Clin Orthop Relat Res 2014;472(08): 2483-2491

18 Grotle M, Hagen KB, Natvig B, Dahl FA, Kvien TK. Obesity and osteoarthritis in knee, hip and/or hand: an epidemiological study in the general population with 10 years follow-up. BMC Musculoskelet Disord 2008;9:132

19 Martin JR, Jennings JM, Dennis DA. Morbid obesity and total knee arthroplasty: a growing problem. J Am Acad Orthop Surg 2017;25 (03):188-194

20 Abdel MP, Bonadurer GF III, Jennings MT, Hanssen AD. Increased aseptic tibial failures in patients with a BMI $\geq 35$ and well-aligned total knee arthroplasties. J Arthroplasty 2015;30(12):2181-2184

21 Culliford D, Maskell J, Judge A, Arden NK; COAST Study group. A population-based survival analysis describing the association of body mass index on time to revision for total hip and knee replacements: results from the UK general practice research database. BMJ Open 2013;3(11):e003614

22 Wallace G, Judge A, Prieto-Alhambra D, de Vries F, Arden NK, Cooper $C$. The effect of body mass index on the risk of postoperative complications during the 6 months following total hip replacement or total knee replacement surgery. Osteoarthritis Cartilage 2014;22(07):918-927

23 Dowsey MM, Choong PF. Obese diabetic patients are at substantial risk for deep infection after primary TKA. Clin Orthop Relat Res 2009;467(06):1577-1581

24 Watts CD, Houdek MT, Wagner ER, Taunton MJ. Subcutaneous fat thickness is associated with early reoperation and infection after total knee arthroplasty in morbidly obese patients. J Arthroplasty 2016;31(08):1788-1791

25 Ledford CK, Millikan PD, Nickel BT, et al. Percent body fat is more predictive of function after total joint arthroplasty than body mass index. J Bone Joint Surg Am 2016;98(10):849-857

26 Fu MC, McLawhorn AS, Padgett DE, Cross MB. Hypoalbuminemia is a better predictor than obesity of complications after total knee arthroplasty: a propensity score-adjusted observational analysis. HSS J 2017;13(01):66-74

27 Walls JD, Abraham D, Nelson CL, Kamath AF, Elkassabany NM, Liu J. Hypoalbuminemia more than morbid obesity is an independent predictor of complications after total hip arthroplasty. J Arthroplasty 2015;30(12):2290-2295

28 Yi PH, Frank RM, Vann E, Sonn KA, Moric M, Della Valle CJ. Is potential malnutrition associated with septic failure and acute infection after revision total joint arthroplasty? Clin Orthop Relat Res 2015;473(01):175-182

29 Werner BC, Evans CL, Carothers JT, Browne JA. Primary total knee arthroplasty in super-obese patients: dramatically higher postoperative complication rates even compared to revision surgery. J Arthroplasty 2015;30(05):849-853

30 Martin JR, Watts CD, Taunton MJ. Bariatric surgery does not improve outcomes in patients undergoing primary total knee arthroplasty. Bone Joint J 2015;97-B(11):1501-1505
31 Maradit Kremers H, Lewallen LW, Mabry TM, Berry DJ, Berbari EF, Osmon DR. Diabetes mellitus, hyperglycemia, hemoglobin A1C and the risk of prosthetic joint infections in total hip and knee arthroplasty. J Arthroplasty 2015;30(03):439-443

32 Marchant MH Jr., Viens NA, Cook C, Vail TP, Bolognesi MP. The impact of glycemic control and diabetes mellitus on perioperative outcomes after total joint arthroplasty. J Bone Joint Surg Am 2009; 91(07):1621-1629

33 Watts CD, Houdek MT, Wagner ER, Abdel MP, Taunton MJ. Insulin dependence increases the risk of failure after total knee arthroplasty in morbidly obese patients. J Arthroplasty 2016;31(01): 256-259

34 Jansen JA, Haddad FS. High prevalence of vitamin D deficiency in elderly patients with advanced osteoarthritis scheduled for total knee replacement associated with poorer preoperative functional state. Ann R Coll Surg Engl 2013;95(08):569-572

35 Maniar RN, Patil AM, Maniar AR, Gangaraju B, Singh J. Effect of preoperative vitamin $D$ levels on functional performance after total knee arthroplasty. Clin Orthop Surg 2016;8(02):153-156

36 Maier GS, Horas K, Seeger JB, Roth KE, Kurth AA, Maus U. Is there an association between periprosthetic joint infection and low vitamin D levels? Int Orthop 2014;38(07):1499-1504

37 Rasouli MR, Menendez ME, Sayadipour A, Purtill JJ, Parvizi J. Direct cost and complications associated with total Joint arthroplasty in patients with preoperative anxiety and depression. J Arthroplasty 2016;31(02):533-536

38 Browne JA, Sandberg BF, D'Apuzzo MR, Novicoff WM. Depression is associated with early postoperative outcomes following total joint arthroplasty: a nationwide database study. J Arthroplasty 2014;29(03):481-483

39 Ali A, Lindstrand A, Sundberg M, Flivik G. Preoperative anxiety and depression correlate with dissatisfaction after total knee arthroplasty: a prospective longitudinal cohort study of 186 patients, with 4-year follow-up. J Arthroplasty 2017;32(03): 767-770

40 Singh JA, Lewallen DG. Depression in primary TKA and higher medical comorbidities in revision TKA are associated with suboptimal subjective improvement in knee function. BMC Musculoskelet Disord 2014;15:127

41 Schwartz FH, Lange J. Factors that affect outcome following total joint arthroplasty: a review of the recent literature. Curr Rev Musculoskelet Med 2017;10(03):346-355

42 Klement MR, Nickel BT, Penrose CT, et al. Psychiatric disorders increase complication rate after primary total knee arthroplasty. Knee 2016;23(05):883-886

43 Gallo J, Goodman SB, Konttinen YT, Raska M. Particle disease: biologic mechanisms of periprosthetic osteolysis in total hip arthroplasty. Innate Immun 2013;19(02):213-224

44 Münch HJ, Jacobsen SS, Olesen JT, et al. The association between metal allergy, total knee arthroplasty, and revision: study based on the Danish Knee Arthroplasty Register. Acta Orthop 2015;86 (03):378-383

45 Granchi D, Cenni E, Giunti A, Baldini N. Metal hypersensitivity testing in patients undergoing joint replacement: a systematic review. J Bone Joint Surg Br 2012;94(08):1126-1134

46 Caicedo MS, Solver E, Coleman L, Jacobs JJ, Hallab NJ. Females with unexplained joint pain following total joint arthroplasty exhibit a higher rate and severity of hypersensitivity to implant metals compared with males: implications of sex-based bioreactivity differences. J Bone Joint Surg Am 2017;99(08):621-628 